\title{
THE PRINCIPAL FIBRATION SEQUENCE AND THE SECOND COHOMOTOPY SET
}

\author{
LAURENCE R. TAYLOR
}

\begin{abstract}
Let $p: E \rightarrow B$ be a principal fibration with classifying map $w: B \rightarrow$ $C$. It is well-known that the group $[X, \Omega C]$ acts on $[X, E]$ with orbit space the image of $p_{\#}$, where $p_{\#}:[X, E] \rightarrow[X, B]$. The isotropy subgroup of the map of $X$ to the base point of $E$ is also well-known to be the image of $[X, \Omega B]$. The isotropy subgroups for other maps $e: X \rightarrow E$ can definitely change as $e$ does.

The set of homotopy classes of lifts of $f$ to the free loop space on $B$ is a group. If $f$ has a lift to $E$, the set $p_{\#}^{-1}(f)$ is identified with the cokernel of a natural homomorphism from this group of lifts to $[X, \Omega C]$.

As an example, $\left[X, S^{2}\right]$ is enumerated for $X$ a 4 -complex.
\end{abstract}

\section{Results And Discussion}

For based spaces $X$ and $Y$, let $[X, Y]$ denote the set of based homotopy classes of maps from $X$ to $Y$. The constant map to the base point makes $[X, Y]$ into a based set. If $Y$ is based, the constant path at the base point is a base point for the based loops, $\Omega Y$.

A principal fibration $p: E \rightarrow B$ is a fibration with a classifying map $w: B \rightarrow C$ such that $E$ is a pull-back of the path-loop fibration for $C$ along $w$. Pick a base point $*_{E} \in E$. Let the base point in $B$ be $*_{B}=p\left(*_{E}\right)$ and let the base point in $C$ be $*_{C}=w\left(*_{B}\right)$, so that $w$ and $p$ become based maps.

It is a result going back to Peterson [6. Lemma 2.1, p. 199] and Nomura [5, Corollary 2.1, p. 118] that there is an exact sequence of based sets

$$
\cdots \rightarrow[X, \Omega B] \rightarrow[X, \Omega C] \rightarrow[X, E] \stackrel{p_{\#}}{\longrightarrow}[X, B] \stackrel{w_{\#}}{\longrightarrow}[X, C]
$$

in the following sense. Each map is a map of based sets and the image of one map is the inverse image of the base point for the following map.

One way to derive this sequence is to fix an $f: X \rightarrow B$ and consider the set $\operatorname{Lift}_{*}^{f}(X \cdots \cdots, E)$ of based homotopy classes of lifts of $f$. There is a forgetful map $\operatorname{Lift}_{*}^{f}(X \cdots \cdots, E) \rightarrow[X, E]$ and properties of fibrations imply that the image is $p_{\#}^{-1}(f)$.

Peterson and Thomas [7, Lemma 4.1, p. 17] show that there is a left action of the group $[X, \Omega C]$ on the set $[X, E]$ which identifies the orbit space with $w_{\#}^{-1}(*)$ where $* \in[X, C]$ is the base point. They show that the set $\operatorname{Lift}_{*}^{f}(X \cdots \cdots, E)$ is a left $[X, \Omega C]$ torsor and this gives exactness in (1.1) at $[X, \Omega C]$. It further follows that the isotropy subgroup of the action on the base point of $[X, E]$ is the image of $[X, \Omega B]$. 
There is another way to proceed. For a space $Y$, let $L Y$ denote the free loop space and let $\epsilon: L Y \rightarrow Y$ denote the projection. The map $\epsilon$ is a fibration. The constant loop at $y \in Y$ defines a section $s: Y \rightarrow L Y$ so the set of lifts has a base point, $s \circ f$. If $*_{Y} \in Y$ is a base point, the space $L Y$ has a base point, $s\left(*_{Y}\right)$. Let $\operatorname{Lift}_{*}^{f}(X \cdots \cdots, L Y)$ denote the set of based homotopy classes of lifts of $f$.

Addition of loops makes $\operatorname{Lift}_{*}^{f}(X \cdots \rightarrow L Y)$ into a group. A based map $w: B \rightarrow$ $C$ induces a based map $L w: L B \rightarrow L C$ and a family of group homomorphisms

$$
\operatorname{Lift}_{*}^{f}(X \cdots L B) \stackrel{\mathcal{L} w_{\#}}{\longrightarrow} \operatorname{Lift}_{*}^{g \circ f}(X \cdots L C)
$$

Theorem 1.2. The set $\operatorname{Lift}_{*}^{f}(X \cdots \cdots, E)$ is a right Lift L $_{*}^{w \circ f}(X \cdots \cdots, C)$ torsor. To each element $e \in[X, E]$ lifting $f \in[X, B]$, there is associated a group isomorphism

$$
\operatorname{Lift}_{*}^{w \circ f}(X \rightarrow L C) \stackrel{\bar{\gamma}_{e}}{\longrightarrow}[X, \Omega C]
$$

The image of the composition

$$
\operatorname{Lift}_{*}^{f}(X \rightarrow L B) \stackrel{\mathcal{L} w_{\#}}{\longrightarrow} \operatorname{Lift}_{*}^{w \circ f}(X \cdots, L C) \stackrel{\bar{\gamma}_{e}}{\longrightarrow}[X, \Omega C]
$$

is the isotropy subgroup of e under the left $[X, \Omega C]$ action on $[X, E]$.

Theorem 1.2 gives a sequence with many of the same properties as (1.1). The group $[X, \Omega C]$ is the same for both sequences. The group $\operatorname{Lift}_{*}^{f}(X \cdots \cdots, B B)$ depends on $B, f$ and $X$ instead of just $X$ and $\Omega B$ as in (1.1), but it is still independent of $C$ and $w$. The homomorphism between these two groups can depend on $f$ in addition to just $w$ (see 46.3 ). The additional information supplied by Theorem 1.2 comes from the fact that all the isotropy subgroups are determined rather than just the isotropy subgroup of the null homotopic map as in (1.1).

Remark 1.3. J. Rutter 9] has results similar to these if $B$ and $C$ are $\mathrm{H}$-spaces. In this case the multiplication can be used to naturally identify $\operatorname{Lift}_{*}^{f}(X \cdots \cdots, L B)$ with $[X, \Omega B]$. Rutter uses the H-space structure to describe a homomorphism $[X, \Omega B] \rightarrow[X, \Omega C]$, depending on $f$, which presumably is related to the homomorphism given by Theorem 1.2 whenever $B$ is an $\mathrm{H}$-space. In general this homomorphism can not be the one induced by $\Omega w$ since the size of the cokernel can vary with $f$. (See 6.9 and 6.10)

The observation that there is a natural right action of the section groups adds generality, and perhaps clarity, to the result.

An additional observation is that the calculations required by (1.1) and Theorem 1.2 are natural in both the space $X$ and the principal fibration.

\section{ReCAll of SOME BASiC RESUlts}

The sequence (1.1) can be derived from standard results about the path-groupoid applied to function spaces. The needed results are recalled below. To prove Theorem 1.2 requires an additional technical lemma, Lemma 2.4 . 
2.1. Point set topology. As usual all constructions take place in a "convenient category", $\mathcal{K}$. Vogt 12 is a good reference. One key point is that the exponential correspondence holds, the space of maps $X \times Y$ to $W$, is homeomorphic to the space of maps of $X$ to $W^{Y}$. Here the product gets the product topology in $\mathcal{K}$ and $W^{Y}$ gets the topology given by starting with the compact-open topology and making it compactly-generated. Also, the subspace topology on a subset is the one given by taking the usual subspace topology and then making it compactly-generated.

If $W_{0} \subset W$ is a subspace, in the category $\mathcal{K}$, then $W_{0}{ }^{Y}$ with its topology is a subspace of $W^{Y}$ with its topology.

Given any point $w \in W$ and any space $Y$, let $\mathfrak{c}_{Y->w} \in W^{Y}$ denote the constant map of $Y$ to $w$. Anytime $W$ has a base point $*_{W} \in W$, the map $\mathfrak{c}_{Y->*}$ will be the base point in $W^{Y}$. If both $Y$ and $W$ are based, then $W_{*}^{Y}$ is the subspace of $W^{Y}$ consisting of all maps $f: Y \rightarrow W$ which preserve the base points.

A base point is non-degenerate provided the pair $\left(W, *_{W}\right)$ is an NDR pair.

If $\left(W, W_{0}\right)$ and $\left(Y, Y_{0}\right)$ are pairs, $\left(W, W_{0}\right)^{\left(Y, Y_{0}\right)}$ denotes the space of all continuous functions $Y \rightarrow W$ sending $Y_{0} \rightarrow W_{0}$. It is given the subspace topology in $\mathcal{K}$ from $W^{Y}$.

Result 2.1. If $\left(W, W_{0}\right)$ is an NDR pair and if $Y$ is compact, then $W_{0}{ }^{Y}$ is a subspace of $\left(W, W_{0}\right)^{\left(Y, Y_{0}\right)}$ and the pair is an NDR pair.

Proof. Since a subspace of a subspace is a subspace $W_{0}{ }^{Y}$ is a subspace of $\left(W, W_{0}\right)^{\left(Y, Y_{0}\right)}$.

If $u: W \rightarrow[0,1]$ is the map which is part of the definition of an NDR pair, then $\hat{u}: W^{Y} \rightarrow[0,1]$ defined by $\hat{u}(f)=\sup _{y \in Y} u(f(y))$ is continuous. This uses $Y$ compact. Note $\hat{u}^{-1}(0)=W_{0}{ }^{Y}$.

If $F: W \times[0,1] \rightarrow W$ is the homotopy which is the other part of the definition of an NDR pair, then if $\hat{F}: W^{Y} \times[0,1] \rightarrow W^{Y}$ is defined by $(\hat{F}(f, t))(y)=F(f(y), t)$, the pair $(\hat{F}, \hat{u})$ shows the function spaces form an NDR pair.

Remark 2.2. If $*_{W} \in W$ is non-degenerate then $\mathfrak{c}_{Y->*}$ is a non-degenerate point in both $W^{Y}$ and $W_{*}^{Y}$.

2.2. The path groupoid. Given two points $w_{0}, w_{1} \in W$ let $W_{w_{0}, w_{1}}$ denote the set of homotopy classes of paths from $w_{0}$ to $w_{1}$ where the homotopies are rel end points. The set $W_{w_{0}, w_{1}}$ is non-empty if and only if $w_{0}$ and $w_{1}$ are in the same path component of $W$.

If $w_{0}, w_{1}$ and $w_{2}$ are all in one path component of $W$, path concatenation defines an associative pairing

$$
W_{w_{0}, w_{1}} \times W_{w_{1}, w_{2}} \rightarrow W_{w_{0}, w_{2}}
$$

Reversing the path defines an involution $W_{w_{0}, w_{1}} \stackrel{-1}{\longrightarrow} W_{w_{1}, w_{0}}$, and hence a bijection, such that the image of the composition $W_{w_{0}, w_{1}} \stackrel{1 \times^{-1}}{\longrightarrow} W_{w_{0}, w_{1}} \times W_{w_{1}, w_{0}} \rightarrow$ $W_{w_{0}, w_{0}}$ is the constant path at $w_{0}$. There is a similar constant map $W_{w_{0}, w_{1}} \rightarrow$ $W_{w_{1}, w_{1}}$.

For any $w \in W, W_{w, w}$ is a group under path concatenation with ${ }^{-1}$ being the inverse map.

If $W_{w_{0}, w_{1}}$ is non-empty, the group $W_{w_{0}, w_{0}}$ acts on it on the left and the group $W_{w_{1}, w_{1}}$ acts on the right. Associativity of path concatenation makes $W_{w_{0}, w_{1}}$ into a bi-set. 
Result 2.3. If non-empty, the bi-set $W_{w_{0}, w_{1}}$ is a torsor for each group action.

Proof. To be a torsor means the group action is transitive and the isotropy subgroup of any point is trivial.

Let $\tau_{0}, \tau_{1} \in W_{w_{0}, w_{1}}$. Then $\tau_{1}=\tau_{0} \bullet\left(\tau_{0}^{-1} \bullet \tau_{1}\right)$ and $\tau_{0}^{-1} \bullet \tau_{1} \in W_{w_{1}, w_{1}}$. Similarly $\tau_{1}=\left(\tau_{1} \bullet \tau_{0}^{-1}\right) \bullet \tau_{0}$ and $\tau_{1} \bullet \tau_{0}^{-1} \in W_{w_{0}, w_{0}}$. Hence both actions are transitive.

Now suppose $\lambda \bullet \tau=\tau$ for some $\lambda \in W_{w_{0}, w_{0}}$ and some $\tau \in W_{w_{0}, w_{1}}$. Then $(\lambda \bullet \tau) \bullet \tau^{-1}=\tau \bullet \tau^{-1}$ and therefore $\lambda$ is homotopic rel end points to the constant path and so the isotropy subgroup of $\tau$ under the left action is trivial. A similar calculation shows the right action also has trivial isotropy subgroups.

Lemma 2.4. Let $\tau_{0}, \tau_{1}$ be representatives of elements in $W_{w_{0}, w_{1}}$ and let $\phi \in$ $W_{w_{1}, w_{1}}$. There exists a homotopy

$$
F:[0,1] \times[0,1] \rightarrow W
$$

with $F(t, 0)=\tau_{0}(t), F(t, 1)=\tau_{1}(t), F(0, s)=w_{0}=\tau_{0}(0)=\tau_{1}(0)$ and $F(1, s)=$ $\phi(s)$ if and only if $\tau_{1}=\tau_{0} \bullet \phi$.

Proof. Let $H$ be a homotopy rel end points from $\tau_{0} \bullet \phi$ to $\tau_{1}$. Figure 1 is a visual representation for $H$ and Figure 2 is one for $F$. Lemma 2.4 is equivalent to constructing $F$ given $H$ and $H$ given $F$.

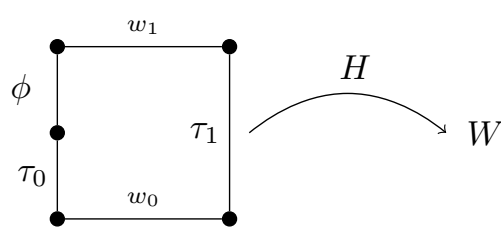

Figure 1

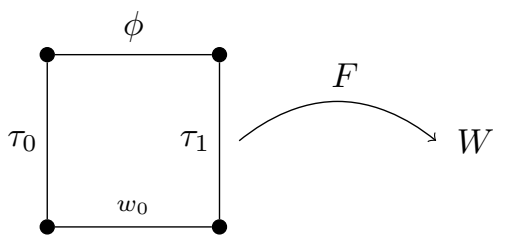

Figure 2

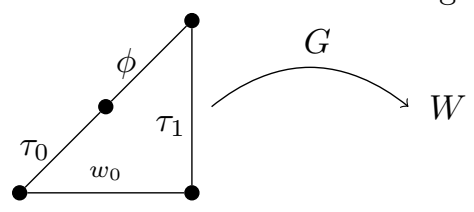

Figure 3

There is an evident map from the squares in Figures 1 and 2 to the triangle in Figure 3. Either map $H$ or $F$ induces a map $G$ from the triangle to $W$. Given $G$, composition with the map from the appropriate square constructs both $F$ and $H$.

2.3. Bi-torsors. Suppose $T$ is a left $G$ torsor and a right $H$ torsor as well as a $G-H$ biset. For $x \in T$, define functions $\gamma_{x}: G \rightarrow H$ and $\bar{\gamma}_{x}: H \rightarrow G$ by $g \bullet x=x \bullet \gamma_{x}(g)$ and $\bar{\gamma}_{x}(h) \bullet x=x \bullet h$.

Result 2.5. Each $x \in T$ defines a group isomorphism $\gamma_{x}: G \rightarrow H$ and an inverse isomorphism $\bar{\gamma}_{x}: H \rightarrow G$

Proof. Note $\gamma_{x}\left(e_{G}\right)=e_{H}$ and $\bar{\gamma}_{x}\left(e_{H}\right)=e_{G}$. Also check $x \bullet \gamma_{x}\left(g_{1} g_{2}\right)=\left(g_{1} g_{2}\right) \bullet x=$ $g_{1} \bullet\left(g_{2} \bullet x\right)=g_{1} \bullet\left(x \bullet \gamma_{x}\left(g_{2}\right)\right)=\left(g_{1} \bullet x\right) \bullet \gamma_{x}\left(g_{2}\right)=x \bullet\left(\gamma_{x}\left(g_{1}\right) \gamma_{x}\left(g_{2}\right)\right)$ so $\gamma_{x}$ is multiplicative. Check $\gamma_{x}$ and $\bar{\gamma}_{x}$ are inverse functions. Hence they are inverse homomorphisms and the result follows. 
Result 2.6. If $x_{1}, x_{2} \in T$, then $\gamma_{x_{1}}$ and $\gamma_{x_{2}}$ are conjugate as are $\bar{\gamma}_{x_{1}}$ and $\bar{\gamma}_{x_{2}}$

Proof. If $x_{1}=x_{2} \bullet h, x_{1} \bullet \gamma_{x_{1}}(g)=g \bullet x_{1}=g \bullet\left(x_{2} \bullet h\right)=x_{2} \bullet\left(\gamma_{x_{2}}(g) h\right)=$ $\left(x_{2} \bullet h\right) \bullet\left(h^{-1} \gamma_{x_{2}}(g) h\right)=x_{1} \bullet\left(h^{-1} \gamma_{x_{2}}(g) h\right)$ so $\gamma_{x_{1}}(g)=h^{-1} \gamma_{x_{2}}(g) h$. The proof for the $\bar{\gamma}$ is similar.

2.4. Principal Fibrations. A principal fibration is a fibration $E \stackrel{p}{\rightarrow} B$ which is a pull-back of the path-loop fibration $\Omega C \rightarrow P C \stackrel{\rho_{C}}{\longrightarrow} C$ along a map $w: B \rightarrow C$. The definition of the space $P C$ requires a base point in $C$, say $*_{C}$. Then $P C$ is the space of all maps $\lambda:[0,1] \rightarrow C$ such that $\lambda(0)=*_{C}$. Equivalently it is the subspace of $C^{[0,1]}$ of paths that start at $*_{C},\left(C, *_{C}\right)^{([0,1], 0)}$.

Up to fibre homotopy equivalence, a principal fibration has a standard model. The total space is $E_{w} \subset B \times C^{[0,1]}$ such that $(b, \lambda) \in E_{w}$ if and only if $w(b)=\lambda(1)$ and $*_{C}=\lambda(0)$. The fibration projection is just projection onto the $B$ factor. If $B$ is given a base point $*_{B}$ such that $w\left(*_{B}\right)=*_{C}$, then $E_{w}$ has a base point, $\left(*_{B}, \mathfrak{c}\right)$ where it should cause no confusion to shorten the notation for the base point in a function space to $\mathfrak{c}$.

For the purposes of this paper it suffices to pick a convenient based map $w$, and then work with $E_{w}$. Two $w$ which are based homotopic yield $E_{w}$ which are based fibre homotopy equivalent and all questions discussed here only depend on the based fibre homotopy type of the fibration.

The next result describes the set of lifts. There is a map induced by composition with $w, w^{X}: B^{X} \rightarrow C^{X}$.

Result 2.7. The set of homotopy classes of lifts of $f \in B^{X}$ is equivalent to the set $W_{w_{0}, w_{1}}$ where $W=C^{X}, w_{0}=\mathfrak{c}$ and $w_{1}=w^{X}(f)$. If $f$ is based, then the set of based homotopy classes of lifts of $f$ is equivalent to the set $W_{w_{0}, w_{1}}$ for the same $w_{i}$ but with $W=C_{*}^{X}$.

Proof. A map of $X$ to $E_{w}$ consists of a map $f: X \rightarrow B$ and a map $\Lambda: X \rightarrow C^{[0,1]}$ satisfying two conditions: $w \circ f(x)=\Lambda(x, 1)$ and $\Lambda(x, 0)=*_{C}$.

Consider the map $f$ as a point $f \in B^{X}$ and the map $\Lambda$ as a map $\Lambda:[0,1] \rightarrow C^{X}$ satisfying two conditions: $\Lambda(0)=\mathfrak{c}$ and $\Lambda(1)=w^{X}(f)$. Two lifts of $f, \Lambda_{0}$ and $\Lambda_{1}$, are homotopic as lifts if and only if $\Lambda_{0}$ and $\Lambda_{1}$ are homotopic rel end-points, that is, they represent the same element in $W_{w_{0}, w_{1}}$.

Result 2.8. Given a map $f \in Y^{X}$, a lift to the free loop space is a map $\Phi:[0,1] \rightarrow$ $Y^{X}$ such that $\Phi(0)=\Phi(1)=f$. In other words, the set of homotopy classes of lifts of $f$ to the free loop space on $Y$ is equivalent to $W_{w, w}$ with $W=Y^{X}$ and $w=f$. If $f \in Y_{*}^{X}$ then the based lifts are equivalent to $W_{w, w}$ with the same $w$ and $W=Y_{*}^{X}$.

\section{The Proof of Theorem 1.2}

Fix a principal fibration $p: E_{w} \rightarrow B, w: B \rightarrow C$. Fix a base point in $B$ and use its image to base $C$. This gives a preferred base point in $E_{w}$. Also fix a based space $X$.

Let $W=C_{*}^{X}$. Since $C$ must have a base point to define $E_{w}$, let $\mathfrak{c}$ be the constant map of $X$ to the base point of $C$. Fix $e: X \rightarrow E$ and let $f=p \circ e$.

Remark 3.1. Given $f: X \rightarrow B$, there exist such $e$ 's if and only if $w \circ f$ is nullhomotopic rel base point. 
Up to homotopy of lifts, $e$ is determined by $f$ and $\Lambda \in W_{\mathfrak{c}, w^{X}(f)}$. The group acting on the left is $W_{\mathfrak{c}, \mathfrak{c}}=[X, \Omega C]$. The group acting on the right is $W_{w^{X}(f), w^{X}(f)}=$ $L i f t_{*}^{w \circ f}(X \cdots \cdots L C)$. The isomorphism $\bar{\gamma}_{e}$ in Theorem 1.2 is the map defined by Result 2.5 .

Two lifts $\Lambda_{0}$ and $\Lambda_{1}$ are homotopic in $[X, E]$ if and only if there are homotopies $\Phi:[0,1] \rightarrow B_{*}^{X}$ with $\Phi(0)=\Phi(1)=f$ and $F:[0,1] \times[0,1] \rightarrow C_{*}^{X}$ such that $F(1, s)=w^{X}(\Phi(s)), F(i, t)=\Lambda_{i}(t), i=0,1$.

Equivalently, $\Phi \in \operatorname{Lift}_{*}^{f}(X \cdots>L B)$ and, if $\phi=w^{X}(\Phi) \in \operatorname{Lift}_{*}^{w \circ f}(X \cdots \cdots L C)$, Lemma 2.4 completes the proof of Theorem 1.2

\section{Some GENERAL REMARKS ON CALCUlations}

There are some situations in which the group of lifts calculation can be replaced by just calculating a set of homotopy classes of maps.

One situation, Corollary 4.2 is a generalization of a result of James and Thomas, [2, Theorem 2.6, p. 493].

Theorem 4.1. Let $Y$ be a based space and let $f: X \rightarrow Y$ be a based map. Then

$$
\left.\mathcal{G}=\operatorname{Lift}_{*}^{f}(X \cdots \rightarrow) L Y\right) \stackrel{\iota}{\longrightarrow}[X, L Y] \stackrel{\epsilon_{\#}}{\longrightarrow}[X, Y]
$$

is exact in that the image of $\iota$ is $\epsilon_{\#}^{-1}(f)$. The image of $\iota$ is also the set of conjugacy classes of elements of $\mathcal{G}$.

Proof. A lift is a map $X \rightarrow L Y=Y^{S^{1}}$. By the exponential correspondence a lift is also a map $S^{1} \rightarrow Y^{X}$. The lift property is equivalent to the additional condition that the base point of $S^{1}$ goes to $f \in Y^{X}$. Hence $\mathcal{G}=\pi_{1}\left(Y^{X} ; f\right)$.

An element $[X, L Y]$ is equal to an element in $\left[S^{1}, Y^{X}\right]$ with no condition on the base points except that the base point of $S^{1}$ lands in the path component of $f$. There is always a homotopy which takes the base point of $S^{1}$ to $f \in Y^{X}$ so the image of $\iota$ is $\epsilon_{\#}^{-1}(f)$.

It is always true that the relation between $\pi_{1}\left(Y^{X} ; f\right)$ and the free homotopy classes is that the set of free homotopy classes is the set of conjugacy classes.

Corollary 4.2 (James \& Thomas, [2]). The group Lift $t_{*}^{f}(X \cdots \cdot L Y$ ) is abelian if and only if $\iota$ is injective.

Given a map $w: B \rightarrow C$, there is an induced map $L w: L B \rightarrow L C$ and

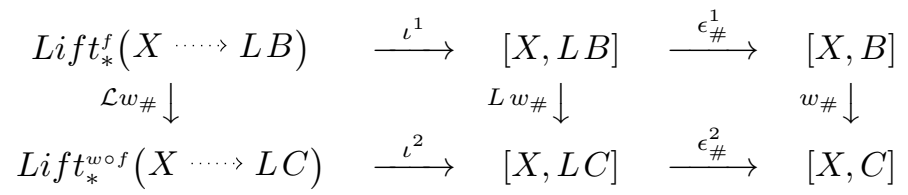

commutes. Hence, if the group $\operatorname{Lift}_{*}^{w \circ f}(X \cdots \cdots, L C)$ is abelian the cokernel of $\mathcal{L} w_{\#}$ can be worked out from knowledge of just the right-hand square. Specifically

Corollary 4.3. With notation as above, suppose Lift wof $_{*}(X \cdots, L C)$ is abelian. The set $\mathcal{C}=\left(\epsilon_{\#}^{2}\right)^{-1}(w \circ f) \subset[X, L C]$ is a group. The set $L w_{\#}\left(\left(\epsilon_{\#}^{1}\right)^{-1}(f)\right)$ is a subgroup of $\mathcal{C}$ and there is a bijection between the coset space of this inclusion and the cokernel of $\mathcal{L} w_{\#}$. 


\section{Some Results ON H-SPACES}

To go further with the analysis in the last section requires some hypotheses. Let $B$ and $C$ be H-spaces which have the homotopy type of CW complexes. Do not assume that the classifying map $w: B \rightarrow C$ is an H-map. Theorem 1.2 under these additional assumptions was obtained by J. W. Rutter [9, Theorem 1.3.1,p. 382] and there is considerable overlap between his $\S 1.4$ and the material here.

If $Y$ has the homotopy type of a $\mathrm{CW}$ complex, so do $L Y$ and $\Omega Y$, see Milnor 4. Theorem 3, p. 276]. If $Y$ is an H-space, the section map $s: Y \rightarrow L Y$ and the inclusion map $\mathrm{i}: \Omega Y \rightarrow L Y$ can be multiplied using the H-space product to give homotopy equivalences, $\Omega Y \times Y \rightarrow L Y$, see James \& Thomas, 2, Theorem 2.7, p. 494], or Zabrodsky, [13, 1.3.6 Proposition, p. 24]. It follows that for any $h: X \rightarrow Y, \operatorname{Lift}_{*}^{h}(X \cdots \cdots, L Y)$ is isomorphic as a group to $[X, \Omega Y]$. Since $Y$ is an H-space, $[X, \Omega Y]$ is abelian and $\left.\operatorname{Lift}_{*}^{h}(X \cdots \cdots) L Y\right)=[X, \Omega Y] \times h \subset[X, \Omega Y] \times$ $[X, Y]=[X, L Y]$.

Hence it suffices to understand $L w_{\#}$ for $w: B \rightarrow C$. If $\alpha \in[X, \Omega B]$ and $\beta \in$ $[X, B]$ write $\alpha \times \beta$ for $\mu_{i}\left(\left(\mathfrak{i}_{i}\right)_{\#}(\alpha),\left(s_{i}\right)_{\#}(\beta)\right)$ Hence, to understand $L w_{\#}$ it suffices to understand $L w_{\#}(\alpha \times \beta)$ where $\alpha \in[X, \Omega B]$ and $\beta \in[X, B]$.

Zabrodsky [13, §1.4, p. 25] discusses the deviation from a map being an H-map. In this case, the deviation is a map $D: L B \wedge L B \rightarrow L C$ which depends on $w$ and is null-homotopic if and only if $w$ is an H-map.

With $\alpha \in[X, \Omega B]$ and $\beta \in[X, B]$ define $W(\alpha, \beta)$ as the composition

$$
X \stackrel{\Delta}{\longrightarrow} X \wedge X \stackrel{\alpha \wedge \beta}{\longrightarrow} \Omega B \wedge B \stackrel{\mathfrak{i}_{1} \wedge \epsilon_{1}}{\longrightarrow} L B \wedge L B \stackrel{D}{\longrightarrow} L C
$$

Then $\mu_{2}\left(W(\alpha, \beta), L w_{\#}(\alpha \times \beta)\right)=(\Omega w)_{\#}(\alpha) \times w_{\#}(\beta)$.

Assume further that $C$ is homotopy-associative so that $L C$ is also homotopyassociative. Then $[X, L C]$ is a group and so

$$
\left.L w_{\#}(\alpha \times \beta)\right)=\mu_{2}\left(W(\alpha, \beta)^{-1},(\Omega w)_{\#}(\alpha) \times w_{\#}(\beta)\right) .
$$

To continue, Zabrodsky [13, 1.4.2 Proposition, p. 25] shows that

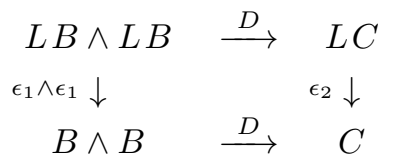

commutes. Hence it follows that the composition $\Omega B \wedge B \stackrel{\mathfrak{i}_{1} \wedge \epsilon_{1}}{\longrightarrow} L B \wedge L B \stackrel{D}{\longrightarrow} L C$ lifts to a map $\Omega B \wedge B \rightarrow \Omega C$. This is a map into $\Omega C$ so it has a multiplicative inverse $\mathfrak{D}: \Omega B \wedge B \rightarrow \Omega C$. Further, for $\alpha \in[X, B]$ and $\beta \in[X, \Omega B]$ define $\alpha \wedge_{w} \beta$ as the composition $X \stackrel{\Delta}{\longrightarrow} X \wedge X \stackrel{\alpha \wedge \beta}{\longrightarrow} \Omega B \wedge B \stackrel{\mathfrak{D}}{\longrightarrow} \Omega C$. Note $\alpha \wedge_{w} \beta$ is bilinear in both $\alpha$ and $\beta$.

Plugging this into the formula above shows $\left.L w_{\#}(\alpha \times \beta)\right)=\mu_{2}\left(\left(\mathfrak{i}_{2}\right)_{\#}\left(\alpha \wedge_{w}\right.\right.$ $\left.\beta),(\Omega w)_{\#}(\alpha) \times w_{\#}(\beta)\right)$. Let $\mu_{3}: \Omega C \times \Omega C \rightarrow \Omega C$ be the usual H-space multiplication and since $\mu_{2}$ is homotopy-associative the next formula has been proved:

(5.1)

$$
\left.L w_{\#}(\alpha \times \beta)\right)=\mu_{3}\left(\left(\alpha \wedge_{w} \beta\right),(\Omega w)_{\#}(\alpha)\right) \times w_{\#}(\beta)
$$

Formula 5.1, Corollary 4.3 and Theorem 1.2 prove 
Theorem 5.2. Let $B$ and $C$ be $H$-spaces with $C$ homotopy-associative. Let $w: B \rightarrow$ $C$ be any map. Let $E$ be the homotopy fibre of $w$, so $\Omega C \rightarrow E \stackrel{p}{\longrightarrow} B$ is a principal fibration. Let $\beta \in[X, B]$ be such that $w_{\#}(\beta)=0$. Then $\left(p_{\#}\right)^{-1}(\beta) \subset[X, E]$ is non-empty and there is a bijection between $\left(p_{\#}\right)^{-1}(\beta)$ and the cokernel of the homomorphism $\psi:[X, \Omega B] \rightarrow[X, \Omega C]$ defined by $\psi(\alpha)=\mu_{3}\left(\left(\alpha \wedge_{w} \beta\right),(\Omega w)_{\#}(\alpha)\right)$ for each $\alpha \in[X, \Omega C]$.

Remark 5.3. Continuing in this vein, let $e \in[X, E]$ be some element with $\mathfrak{p}_{\#}(e)=$ $\beta$. Let

$$
\mathfrak{D}^{\prime}: \Omega B \wedge E \stackrel{1_{\Omega B} \wedge \mathfrak{p}}{\longrightarrow} \Omega B \wedge B \stackrel{\mathfrak{D}}{\longrightarrow} \Omega C
$$

and define $\alpha \wedge_{w}^{\prime} e$ as the composition $X \stackrel{\Delta}{\longrightarrow} X \wedge X \stackrel{\alpha \wedge e}{\longrightarrow} \Omega B \wedge E \stackrel{\mathfrak{D}^{\prime}}{\longrightarrow} \Omega C$. Certainly $\mu_{3}\left(\left(\alpha \wedge_{w} \beta\right),(\Omega w)_{\#}(\alpha)\right)$ and $\mu_{3}\left(\left(\alpha \wedge_{w}^{\prime} e\right),(\Omega w)_{\#}(\alpha)\right)$ have the same image and sometimes $\mathfrak{D}^{\prime}$ is easier to compute than $\mathfrak{D}$.

Further information on $\mathfrak{D}$ can be obtained by applying (5.1) to the identity map which yields the next result.

Theorem 5.4. The composition $\Omega B \times B \longrightarrow L B \stackrel{L w}{\longrightarrow} L C$ is homotopic to the following composition.

$\Omega B \times B \stackrel{\mathbf{s} \times 1}{\longrightarrow}(\Omega B \wedge B) \times(\Omega B \times B) \stackrel{\mathcal{D} \times \Omega w \times w}{\longrightarrow} \Omega C \times \Omega C \times C \longrightarrow \Omega C \times C \longrightarrow L C$ where s: $\Omega B \times B \rightarrow \Omega B \wedge B$ is the usual map.

Corollary 5.5. Suppose $a \in H_{r_{1}}(\Omega B ; \mathbb{Z})$ and $b \in H_{r_{2}}(E ; \mathbb{Z})$ are primitive classes, $r_{i}>0$. Then $\mathfrak{D}_{*}^{\prime}(a \times b) \in H_{r_{1}+r_{2}}(\Omega C ; \mathbb{Z})$ maps to $L w_{*}(a \times b) \in H_{r_{1}+r_{2}}(L C ; \mathbb{Z})$.

Proof. Since both $a$ and $b$ are primitive, the composition

$$
\Omega B \times E \stackrel{\Delta}{\longrightarrow}(\Omega B \times E) \times(\Omega B \times E) \rightarrow(\Omega B \wedge E) \times(\Omega B \times E)
$$

on $a \times b$ is $(a \wedge b) \times(1 \times 1)+1 \times(a \times b)$. By Theorem 5.4 the result follows since $w_{*}(b)=0$.

Remark 5.6. If $C$ is not an H-space but is highly connected, then replace $C$ by $\Omega \Sigma C$ and consider the composition $B \rightarrow C \stackrel{\iota}{\longrightarrow} \Omega \Sigma C$ where $\iota$ is the canonical inclusion. There is a commutative ladder

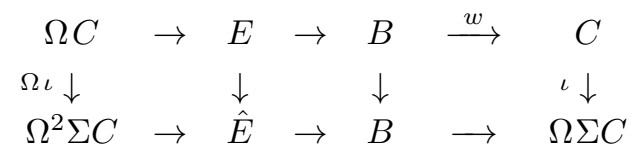

If $\pi_{i}(C)=0$ for $i<n$, then for any complex $X$ of dimension $\leqslant 2 n-2,[X, C] \rightarrow$ $[X, \Omega \Sigma C]$ is an isomorphism as are the other induced vertical maps. The results above can be applied to the $\hat{E}$ principal fibration to yield results about the $E$ principal fibration. 


\section{Some EXAmples}

6.1. Steenrod's problem. Steenrod [11] solved the problem of enumerating the homotopy classes of maps $\left[X, S^{n}\right]$ where $n \geqslant 3$ and $X$ is a CW complex of dimension at most $n+1$. Theorem 1.2 is not needed for the calculations in this subsection, but the results are needed below. A modern approach to this problem goes as follows.

For $n \geqslant 1$, let $S E_{n}$ be the fibre of the map $K(\mathbb{Z}, n) \stackrel{S q^{2}}{\longrightarrow} K(\mathbb{Z} / 2 \mathbb{Z}, n+2)$. There is a map $S^{n} \rightarrow S E_{n}$ and the induced map $\left[X, S^{n}\right] \rightarrow\left[X, S E_{n}\right]$ is an isomorphism if $n \geqslant 3$ and the dimension of $X$ is at most $n+1$. In other words, $S E_{n}$ is the first two stages of a Postnikov decomposition for $S^{n}$. The needed calculations are due to Serre [10.

For $n \geqslant 3, S E_{n}=\Omega S E_{n+1}$ so $S E_{n}$ is a homotopy-abelian H-space, $\left[X, S E_{n}\right]$ is an abelian group, and the fibration de-loops. Write coker $\left(\overline{S q}^{2}\right)$ for the $\mathbb{Z} / 2 \mathbb{Z}$ vector space $H^{n+1}(X ; \mathbb{Z} / 2 \mathbb{Z}) / S q^{2}\left(H^{n-1}(X ; \mathbb{Z})\right)$. Steenrod's main theorem [11, Theorem 28.1, p. 318] follows:

$$
0 \rightarrow \operatorname{coker}\left(\overline{S q}^{2}\right) \rightarrow\left[X, S^{n}\right] \rightarrow H^{n}(X ; \mathbb{Z}) \rightarrow 0
$$

is an exact sequence of abelian groups.

Historically of course this approach is backwards. Steenrod invented $S q^{2}$ to solve this problem and then worked out the Steenrod algebra which led to Serre's work. One could make a case for this being one of the all-time most important problems in algebraic topology.

Larmore and Thomas [3, §5] give a procedure to determine the extension in (6.1). In this case their procedure reduces to determining how the kernel of the multiplication by $2^{k}$ on $H^{n}(X ; \mathbb{Z})$ maps into $\left[X, S^{n}\right]$. To analyze this, consider the $2^{k}$ power maps on $S E_{n}, \mathfrak{s}_{k}, k \geqslant 1$. For each $k$ there is a commutative ladder of fibrations

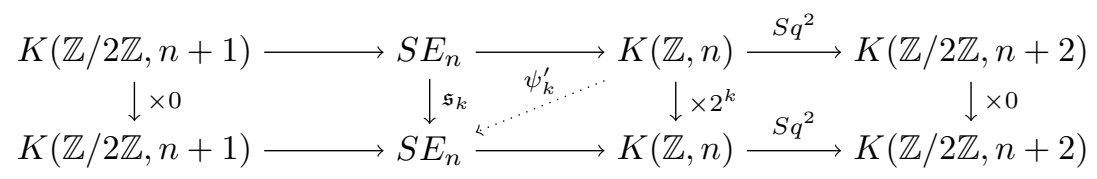

Since the rows are fibrations (up to homotopy) there exists a map $\psi_{k}^{\prime}$ as indicated in the diagram making the lower triangle commute. Since $H^{n+1}\left(K(\mathbb{Z}, n) ; \mathbb{Z} / 2^{k} \mathbb{Z}\right)=$ 0 , the map $\psi^{\prime}$ is unique. It follows from the Serre spectral sequence for the fibration that $H^{n+1}\left(S E_{n} ; \mathbb{Z} / 2^{k} \mathbb{Z}\right)=0$ so the upper triangle involving $\psi_{k}^{\prime}$ also commutes.

Next check that the following diagram commutes.

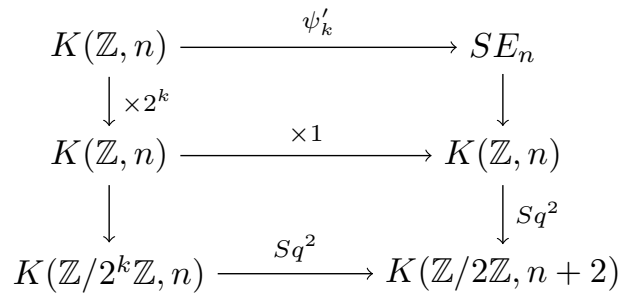

It follows that there is an induced map on the fibres which is the loops of $S q^{2}$ and is therefore again $S q^{2}$. Hence 


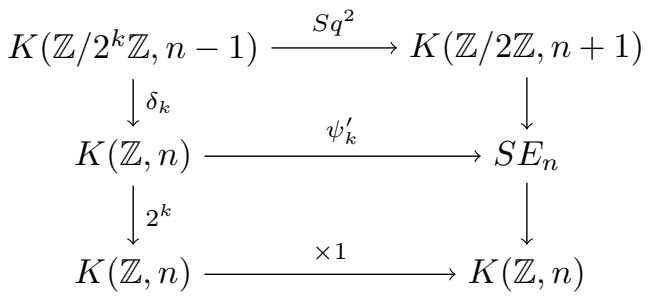

commutes, where $\delta_{k}$ is the evident Bockstein. The next result summarizes the above discussion.

Theorem 6.2. Let $X$ be a finite complex of dimension $\leqslant n+1$. Fix $\gamma \in H^{n}(X ; \mathbb{Z})$ and suppose there is a $k \geqslant 1$ such that $2^{k} \gamma=0$. Pick $\gamma^{\prime} \in H^{n-1}\left(X ; \mathbb{Z} / 2^{k} \mathbb{Z}\right)$ with $\delta_{k}\left(\gamma^{\prime}\right)=\gamma$ and then compute $S q^{2}\left(\gamma^{\prime}\right) \in H^{n+1}(X ; \mathbb{Z} / 2 \mathbb{Z}) / S q^{2}\left(H^{n-1}(X ; \mathbb{Z})\right) \subset$ $\left[X, S^{n}\right]$. For any $\bar{\gamma} \in\left[X, S^{n}\right]$ which maps to $\gamma, 2^{k} \bar{\gamma}=\psi_{k}^{\prime}(\gamma)=S q^{2}\left(\gamma^{\prime}\right)$.

Example 6.3. Suppose $X$ is a complex of dimension $\leqslant n+1$ and suppose that $S q^{2}: H^{n-1}(X ; \mathbb{Z}) \rightarrow H^{n+1}(X ; \mathbb{Z} / 2 \mathbb{Z})$ and $S q^{2}: H^{n-1}(X ; \mathbb{Z} / 2 \mathbb{Z}) \rightarrow H^{n+1}(X ; \mathbb{Z} / 2 \mathbb{Z})$ have the same image. Then $\left[X, S^{n}\right]=\operatorname{coker}\left(\overline{S q}^{2}\right) \oplus H^{n}(X ; \mathbb{Z})$.

Example 6.4. If $X^{4}$ is Habegger's manifold 1 or an Enriqué's surface, then $S q^{2}: H^{2}(X ; \mathbb{Z}) \rightarrow H^{4}(X ; \mathbb{Z} / 2 \mathbb{Z})$ is zero but $S q^{2}: H^{2}(X ; \mathbb{Z} / 2 \mathbb{Z}) \rightarrow H^{4}(X ; \mathbb{Z} / 2 \mathbb{Z})$ is onto. Since $H^{3}(X ; \mathbb{Z})=\mathbb{Z} / 2 \mathbb{Z}$ it follows that $\left[X, S^{3}\right] \cong \mathbb{Z} / 4 \mathbb{Z}$.

6.2. Pontrjagin's problem. Pontrjagin [8] solved the problem of enumerating $\left[X, S^{2}\right]$ for $X$ a 3-complex before Steenrod did his work. From the point of view taken here, $S^{2} \rightarrow \mathbf{B} S^{1} \rightarrow \mathbf{B} S^{3}$ is a fibration so $S^{2}$ is the total space of a principal fibration, $S^{3} \rightarrow S^{2} \rightarrow \mathbf{B} S^{1}$. Since $S^{1}$ is an abelian group, $\mathbf{B} S^{1}=\mathbb{C P}^{\infty}$ is an H-space. However, $S^{3}$ is not abelian and $\mathbf{B} S^{3}=\mathbb{H} \mathbb{P}^{\infty}$ is not an $\mathrm{H}$-space.

However, $\pi_{i}\left(\mathbf{B} S^{3}\right)=0$ for $i<4$ so Remark [5.6] says that as long as the dimension of $X$ is $\leqslant 2 \cdot 4-2=6$, the theorems in $\S 5$ apply. The next subsection computes the answer for all complexes of dimension $\leqslant 4$ and includes a statement and proof of Pontrjagin's result as Corollary 6.9.

6.3. The second cohomotopy set of a 4-complex. Let $X$ have the homotopy type of a $\mathrm{CW}$-complex of dimension $\leqslant 4$. The first step is to compute the map $\left[X, \mathbf{B} S^{1}\right] \rightarrow\left[X, \mathbf{B} S^{3}\right]$. The map $\mathbf{B} S^{3} \rightarrow K(\mathbb{Z}, 4)$ giving a generator of $H^{4}\left(\mathbf{B} S^{3} ; \mathbb{Z}\right) \cong \mathbb{Z}$ is 5 -connected, so $\left[X, \mathbf{B} S^{3}\right] \rightarrow[X, K(\mathbb{Z}, 4)]=H^{4}(X ; \mathbb{Z})$ is an isomorphism. Since the map $\mathbf{B} S^{1} \rightarrow \mathbf{B} S^{3}$ is the standard inclusion of $\mathbb{C P}^{\infty}$ in $\mathbb{H} \mathbb{P}^{\infty}$, the map $\left[X, \mathbf{B} S^{1}\right]=H^{2}(X ; \mathbb{Z}) \rightarrow\left[X, \mathbf{B} S^{3}\right]=H^{4}(X ; \mathbb{Z})$ is just the cup product square. Hence $\left[X, S^{2}\right] \rightarrow H^{2}(X ; \mathbb{Z})$ is onto the subset of classes $\beta \in H^{2}(X ; \mathbb{Z})$ such that $\beta \cup \beta=0 \in H^{4}(X ; \mathbb{Z})$.

Since $\mathbf{B} S^{3}$ is not an H-space, use Remark 5.6 and work with $\Omega \Sigma \mathbf{B} S^{3}$.

In $\S 6.1$, the group $\left[X, S^{3}\right]=\left[X, \Omega^{2} \Sigma \mathbf{B} S^{3}\right]$ was computed for any 4-complex.

For a fixed map $e: X \rightarrow S^{2}$, the next step is to understand the homomorphism $\psi_{e}: H^{1}(X ; \mathbb{Z}) \rightarrow\left[X, \Omega \Sigma \mathbf{B} S^{3}\right]$. Since $\alpha \in H^{1}(X ; \mathbb{Z})$ is equivalent to a homotopy class of based maps $\alpha: X \rightarrow S^{1}$, and since $\mathfrak{D}^{\prime}: S^{1} \wedge S^{2} \rightarrow \Omega^{2} \Sigma \mathbf{B} S^{3}$, it follows that $\mathfrak{D}^{\prime}$ factors through the degree $c_{e}$-map $S^{3} \rightarrow S^{3}$. Hence there is a homomorphism $\bar{\psi}: H^{1}(X ; \mathbb{Z}) \rightarrow\left[X, S^{3}\right]$ such that $\psi_{e}$ is the composition

$$
H^{1}(X ; \mathbb{Z}) \stackrel{\bar{\psi}}{\longrightarrow}\left[X, S^{3}\right] \stackrel{\left(c_{e}\right)_{\#}}{\longrightarrow}\left[X, S^{3}\right]
$$


where $\left(c_{e}\right)_{\#}$ is the map induced by the degree $c_{e}$ map on $S^{3}$. Since $\left[X, S^{3}\right]$ is an abelian group, $\left(c_{e}\right)_{\#}$ is just multiplication by $c_{e}$.

By definition, the composition $H^{1}(X ; \mathbb{Z}) \stackrel{\bar{\psi}}{\longrightarrow}\left[X, S^{3}\right] \rightarrow H^{3}(X ; \mathbb{Z})$ just sends $\alpha$ to $\alpha \cup \beta$ where $\beta \in H^{2}(X ; \mathbb{Z})$ is given by pulling back the fundamental class in $H^{2}\left(S^{2} ; \mathbb{Z}\right)$ via $e: X \rightarrow S^{2}$. It follows from Lemma 6.5 below that $c_{e}= \pm 2$. The sign will not be determined here.

Lemma 6.5. The map $H_{3}\left(L \mathbf{B} S^{1} ; \mathbb{Z}\right) \rightarrow H_{3}\left(L \mathbf{B} S^{3} ; \mathbb{Z}\right)$ is multiplication by \pm 2 .

Proof. For $m=1$ or 3 , the Serre spectral sequence for $S^{m} \rightarrow L \mathbf{B} S^{m} \rightarrow \mathbf{B} S^{m}$ collapses and $H_{*}\left(L \mathbf{B} S^{m} ; \mathbb{Z}\right)=E\left(e_{m}\right) \otimes \mathbb{Z}\left[x_{m+1}\right]$ where $e_{m} \in H_{m}\left(L \mathbf{B} S^{m} ; \mathbb{Z}\right)$ is the image of $H_{m}\left(S^{m} ; \mathbb{Z}\right) ; x_{m+1} \in H_{m+1}\left(L \mathbf{B} S^{m} ; \mathbb{Z}\right)$ maps to a generator of $H_{m+1}\left(\mathbf{B} S^{m} ; \mathbb{Z}\right)$; $E\left(e_{m}\right)$ is an exterior algebra and $\mathbb{Z}\left[x_{m+1}\right]$ is a polynomial algebra.

Now $H_{2}\left(L S^{2} ; \mathbb{Z}\right) \cong \mathbb{Z} \oplus \mathbb{Z} / 2 \mathbb{Z}$, say by Ziller, [14, p. 21]. It follows that in the Serre spectral sequence for the fibration $L S^{2} \rightarrow L \mathbf{B} S^{1} \rightarrow L \mathbf{B} S^{3}$ there is a single differential from $H_{3}\left(L \mathbf{B} S^{3} ; \mathbb{Z}\right)$ onto $\mathbb{Z} / 2 \mathbb{Z}$ so $H_{3}\left(L \mathbf{B} S^{1} ; \mathbb{Z}\right) \rightarrow H_{3}\left(L \mathbf{B} S^{3} ; \mathbb{Z}\right)$ is multiplication by \pm 2 .

It follows that the homomorphism $\psi_{e}: H^{1}(X ; \mathbb{Z}) \rightarrow\left[X, S^{3}\right]$ factors as

$$
H^{1}(X ; \mathbb{Z}) \stackrel{(-) \cup \beta}{\longrightarrow} H^{3}(X ; \mathbb{Z}) \stackrel{\psi_{1}^{\prime}}{\longrightarrow}\left[X, S^{3}\right]
$$

and so $\psi_{e}$ only depends on $\beta$ and hereafter will be written $\psi_{\beta}$

Theorem 6.6. Let $X$ be a complex of dimension $\leqslant 4$ and let $p_{\#}:\left[X, S^{2}\right] \rightarrow$ $H^{2}(X ; \mathbb{Z})$ be the map pulling back a fixed generator of $H^{2}\left(S^{2} ; \mathbb{Z}\right)$.

If $\beta \in H^{2}(X ; \mathbb{Z})$ is given, then $p_{\#}^{-1}(\beta)$ is non-empty if and only if $\beta \cup \beta=0$. Furthermore, if $p_{\#}^{-1}(\beta)$ is non-empty, then there is a bijection between it and the cokernel of $\psi_{\beta}: H^{1}(X ; \mathbb{Z}) \rightarrow\left[X, S^{3}\right]$.

Remark 6.7. Let $P_{\beta}$ be the cokernel of $H^{1}(X ; \mathbb{Z}) \stackrel{2\left({ }_{-}\right) \cup \beta}{\longrightarrow} H^{3}(X ; \mathbb{Z})$. Then there is an exact sequence

$$
\operatorname{coker}\left(\overline{S q}^{2}\right) \stackrel{q}{\longrightarrow} \operatorname{coker}\left(\psi_{\beta}\right) \rightarrow P_{\beta} \rightarrow 0
$$

The kernel of $q$ is the set of all elements of the form $S q^{2}(a)$ for some $a \in H^{2}(X ; \mathbb{Z} / 2 \mathbb{Z})$ such that there exists $\alpha \in H^{1}(X ; \mathbb{Z})$ such that $\delta_{1}(a)=\alpha \cup \beta \in H^{3}(X ; \mathbb{Z})$.

Remark 6.8. There are three types of connected, closed, compact 4-manifolds: (1) there exists an $x \in H^{2}(X ; \mathbb{Z})$ with odd square; (2) for all $x \in H^{2}(X ; \mathbb{Z} / 2 \mathbb{Z}) x \cup x=0$; (3) $X$ is not of type (2) but for all $x \in H^{2}(X ; \mathbb{Z}) x \cup x$ is even. If $X$ has type (1), coker $\left(\psi_{\beta}\right) \rightarrow P_{\beta}$ is an isomorphism. If $X$ has type $(2) 0 \rightarrow \mathbb{Z} / 2 \mathbb{Z} \rightarrow \operatorname{coker}\left(\psi_{\beta}\right) \rightarrow$ $P_{\beta} \rightarrow 0$ is split exact. If $X$ has type (3) $\mathbb{Z} / 2 \mathbb{Z} \rightarrow \operatorname{coker}\left(\psi_{\beta}\right) \rightarrow P_{\beta} \rightarrow 0$ is exact and Theorem 6.2 can be used to determine the group. If $X$ has type (3) and if coker $\left(\psi_{\beta}\right) \rightarrow P_{\beta}$ is not an isomorphism, then the sequence is not split. The manifold $\mathbb{C P}^{2}$ has type (1), any Spin manifold has type (2) and the Habegger manifold [1] is an example a type (3) manifold for which the extension is not split. The author does not know an example of a type (3) manifold for which coker $\left(\psi_{\beta}\right) \rightarrow P_{\beta}$ is an isomorphism.

Corollary 6.9 (Pontrjagin, [8]). If $X$ is a 3-dimensional complex then $\left[X, S^{2}\right] \rightarrow$ $H^{2}(X ; \mathbb{Z})$ is onto and there is a bijection between $p_{\#}^{-1}(\beta)$ and $P_{\beta}$. 
Example 6.10. Let $X=S^{2} \times S^{1}$. Then $H^{2}(X ; \mathbb{Z}) \cong \mathbb{Z}$ : let $\gamma$ be a generator. If $\beta=c \gamma$ then there are maps $X \rightarrow S^{2}$ such that $\beta$ is the image of a generator of $H^{2}\left(S^{2} ; \mathbb{Z}\right)$ and there is a bijection between $p_{\#}^{-1}(\beta)$ and $\mathbb{Z}$ if $c=0$ and $\mathbb{Z} / 2 c \mathbb{Z}$ otherwise.

Example 6.11. Let $X=S^{2} \times S^{1} \times S^{1}$. Let $\left\{\mathfrak{a}_{1}, \mathfrak{a}_{2}\right\} \subset H^{1}(X ; \mathbb{Z}) \cong \mathbb{Z} \oplus \mathbb{Z}$ be a basis and let $\left\{\mathfrak{a}=\mathfrak{a}_{1} \cup \mathfrak{a}_{2}, \mathfrak{b}\right\} \subset H^{2}(X ; \mathbb{Z})$ be a basis. It follows that $\left\{\mathfrak{b} \cup \mathfrak{a}_{1}, \mathfrak{b} \cup \mathfrak{a}_{2}\right\}$ is a basis for $H^{3}(X ; \mathbb{Z})$. Then $\beta=a \mathfrak{a}+b \mathfrak{b}$ has square 0 if and only if $a \cdot b=0$. If $b=0$, then coker $\left(\psi_{\beta}\right)=H^{3}(X ; \mathbb{Z}) \oplus \mathbb{Z} / 2 \mathbb{Z} \cong \mathbb{Z}^{2} \oplus \mathbb{Z} / 2 \mathbb{Z}$. If $a=0$, then the image of $\psi_{\beta}$ is spanned by $(2 b) \mathfrak{b} \cup \mathfrak{a}_{1}$ and $(2 b) \mathfrak{b} \cup \mathfrak{a}_{2}$ and so $\operatorname{coker}\left(\psi_{\beta}\right) \cong \mathbb{Z} / 2 b \mathbb{Z} \oplus \mathbb{Z} / 2 b \mathbb{Z} \oplus \mathbb{Z} / 2 \mathbb{Z}$.

\section{REFERENCES}

[1] Nathan Habegger, Une variété de dimension 4 avec forme d'intersection paire et signature -8, Comment. Math. Helv. 57 (1982), no. 1, 22-24 (French). MR 672843 (83k:57018)

[2] Ioan James and Emery Thomas, An approach to the enumeration problem for non-stable vector bundles, J. Math. Mech. 14 (1965), 485-506. MR 0175134 (30 \#5319)

[3] Lawrence L. Larmore and Emery Thomas, Group extensions and principal fibrations, Math. Scand. 30 (1972), 227-248. MR 0328935 (48 \#7277)

[4] John Milnor, On spaces having the homotopy type of a CW-complex, Trans. Amer. Math. Soc. 90 (1959), 272-280. MR 0100267 (20 \#6700)

[5] Yasutoshi Nomura, On mapping sequences, Nagoya Math. J. 17 (1960), 111-145. MR 0132545 (24 \#A2385)

[6] Franklin P. Peterson, Functional cohomology operations, Trans. Amer. Math. Soc. 86 (1957), 197-211. MR 0105679 (21 \#4417)

[7] Franklin P. Peterson and Emery Thomas, A note on non-stable cohomology operations, Bol. Soc. Mat. Mexicana (2) 3 (1958), 13-18. MR 0105680 (21 \#4418)

[8] Lev Semenovich Pontrjagin, A classification of mappings of the three-dimensional complex into the two-dimensional sphere, Rec. Math. [Mat. Sbornik] N. S. 9 (51) (1941), 331-363 (English, with Russian summary). MR 0004780 (3,60g)

[9] John W. Rutter, A homotopy classification of maps into an induced fibre space, Topology 6 (1967), 379-403. MR 0214070 (35 \#4922)

[10] Jean-Pierre Serre, Cohomologie modulo 2 des complexes d'Eilenberg-MacLane, Comment. Math. Helv. 27 (1953), 198-232 (French). MR 0060234 (15,643c)

[11] Norman E. Steenrod, Products of cocycles and extensions of mappings, Ann. of Math. (2) 48 (1947), 290-320. MR 0022071 (9,154a)

[12] Rainer M. Vogt, Convenient categories of topological spaces for homotopy theory, Arch. Math. (Basel) 22 (1971), 545-555. MR 0300277 (45 \#9323)

[13] Alexander Zabrodsky, Hopf spaces, North-Holland Publishing Co., Amsterdam, 1976. North-Holland Mathematics Studies, Vol. 22; Notas de Matemática, No. 59. MR 0440542 (55 \#13416)

[14] Wolfgang Ziller, The free loop space of globally symmetric spaces, Invent. Math. 41 (1977), no. 1, 1-22. MR 0649625 (58 \#31198)

Department of Mathematics, University of Notre Dame, Notre Dame, IN 46556

E-mail address: taylor.2@nd.edu 